\title{
Sequence of the haemagglutinin-neuraminidase gene of the Newcastle disease virus oral vaccine strain V4(UPM)
}

\begin{abstract}
The nucleotide sequence of the haemagglutinin-neuraminidase (HN) glycoprotein gene of Newcastle disease virus (NDV) variant strain V4(UPM) was determined by direct genomic RNA sequencing and confirmed by cycle sequencing. The gene comprises 1996 nucleotides encoding a 615 amino acid protein of size $67.4 \mathrm{kDa}$. The nucleotide and amino acid sequences of this strain were compared with those of the parent strain V4(QUE). There are 16 nucleotide substitutions on V4(UPM), eight of which are silent mutations and another eliminated a potential Asn-linked glycosylation site in V4(UPM). In addition, an Arg (403) residue was shown to be absent in the variant strain. This deletion is thought to be significant because of its location in a highly conserved region of the HN protein.
\end{abstract}

Keyword: Haemagglutinin-neuraminidase (HN); Newcastle disease virus (NDV); V4(UPM) 